\title{
Clinical factors predicting blood pressure reduction after catheter-based renal denervation
}

\author{
Krzysztof Bartus ${ }^{1}$, Radoslaw Litwinowicz ${ }^{1}$, Jerzy Sadowski ${ }^{1}$, Wojciech Zajdel ${ }^{2}$, Maciej Brzezinski ${ }^{3}$, \\ Magdalena Bartus ${ }^{4}$, Paweł Kleczyński ${ }^{5}$, Stanislaw Bartus ${ }^{6}$, Dhanunjaya Lakkireddy, Boguslaw Kapelak ${ }^{1}$
}

\begin{abstract}
${ }^{1}$ Department of Cardiovascular Surgery and Transplantology, Jagiellonian University Medical College, John Paul II Hospital, Krakow, Poland ${ }^{2}$ Department of Interventional Cardiology, John Paul II Hospital, Jagiellonian University Medical College, Krakow, Poland ${ }^{3}$ Department of Cardiac and Vacsular Surgery, Medical University of Gdansk, Gdansk, Poland ${ }^{4}$ Department of Experimental Pharmacology, Jagiellonian University Medical College, Krakow, Poland ${ }^{5} 2^{\text {nd }}$ Department of Cardiology, Institute of Cardiology, Jagiellonian University Medical College, Krakow, Poland ${ }^{6}$ Department of Interventional Cardiology, Institute of Cardiology, Jagiellonian University Medical College, Krakow, Poland ${ }^{7}$ Division of Cardiovascular Diseases, Cardiovascular Research Institute, University of Kansas, Kansas City, USA
\end{abstract}

Adv Interv Cardiol 2018; 14, 3 (53): 270-275

DOI: https://doi.org/10.5114/aic.2018.78330

\begin{abstract}
Introduction: Renal denervation (RD) can lead to a significant and sustained decrease in mean values of arterial blood pressure (BP). However, there is still a subset of patients without a significant BP drop after RD (non-responders).

Aim: To compare characteristics of RD responders to RD non-responders and to identify the clinical predictors of BP reduction.

Material and methods: Thirty-one patients with diagnosed resistant hypertension underwent RD. Three years after RD the analysis of $\mathrm{BP}$ reduction was performed in regard to the baseline patient characteristics.

Results: After 3 years' follow-up a 10\% or more reduction of systolic baseline BP was observed in $74 \%$ of patients. Ten percent or more reduction of diastolic baseline BP was observed in $71 \%$ of patients. Among responders we observed the following risk factors: hypercholesterolemia in $70 \%$, body mass index (BMI) $>30 \mathrm{~kg} / \mathrm{m}^{2}$ in $55 \%$, diabetes mellitus in $35 \%$, current smoking in $5 \%$. Comorbidity included coronary artery disease (CAD) in 30\%, cardiomyopathy in 10\%, chronic obstructive pulmonary disease (COPD) in $10 \%$, renal insufficiency in $10 \%$, and ventricular arrhythmia in $5 \%$. Among non-responders we observed the following risk factors: hypercholesterolemia in $38 \%$, diabetes mellitus type 2 in $38 \%$ and $\mathrm{BMI}>30 \mathrm{~kg} / \mathrm{m}^{2}$ in $86 \%$. Comorbidity included CAD in $50 \%$ and cardiomyopathy in $13 \%$ of patients.

Conclusions: A $10 \%$ reduction of systolic baseline BP was observed in $74 \%$ of patients 3 years after renal denervation. Clinical factors like COPD, chronic kidney disease 3a, female sex and hypercholesterolemia increase the chances of effective reduction of BP.
\end{abstract}

Key words: hypertension, resistant hypertension, renal denervation.

S u m m a ry

Catheter-based renal nerve ablation with radiofrequency energy via the renal artery can lead to significant and sustained decrease in mean values of arterial systolic and diastolic blood pressure (BP) in patients with resistant hypertension. However, there is still a large group of non-responding patients, without significant blood pressure changes after renal denervation (RD). The reason of non-responsiveness to RD remains still to be clarified. The aim of the study was to retrospectively compare RD responders patients to RD non-responders and find predictors of non-responsiveness for RD as well as clinical factors that might have direct impact on the blood pressure reduction to increase the chances of procedural success. Three years after procedure, mean BP value remained successfully reduced (> 10\% reduction of baseline BP) in $74 \%$ of systolic BP and in $71 \%$ of diastolic BP. Clinical factors like chronic obstructive pulmonary disease, chronic kidney disease 3a, female sex and hypercholesterolemia increase the chances of effective reduction of BP.

\section{Introduction}

Catheter-based renal nerve ablation with radiofrequency energy via the renal artery can lead to a signif- icant and sustained decrease in mean values of arterial systolic and diastolic blood pressure (BP) in patients with resistant hypertension (systolic $\mathrm{BP}<160 \mathrm{~mm} \mathrm{Hg}$ ). The

\section{Corresponding author:}

Stanislaw Bartus, Department of Interventional Cardiology, Institute of Cardiology, Jagiellonian University Medical College, Krakow, Poland, phone: +48 1242471 70, fax: +48 1242471 84, e-mail: sbartus@cathlab.krakow.pl

Received: 5.10.2017, accepted: 4.06.2018. 
first procedure of renal denervation (RD) was performed by Krum et al. in 2009 [1]. The clinical trials Symplicity I and Symplicity II have shown that $84 \%$ of patients after RD had a significant decrease of BP, reaching mean systolic and diastolic depression by 30 and $14 \mathrm{~mm} \mathrm{Hg}$ $[1,2]$. Effective BP lowering therapy can lead to several subsequent benefits including reduction of cardiovascular mortality and morbidity [1-4] but also improvement of quality of life [5]. However, there is still a large group of non-responding patients, without significant blood pressure changes after RD. The first short-term results showed that $84 \%$ of the patients had BP $\geq 10 \mathrm{~mm} \mathrm{Hg}$ and $39 \%$ had desirable $\mathrm{BP}(\leq 140 \mathrm{~mm} \mathrm{Hg}$ ) [1]. The reason for non-responsiveness to RD still remains to be clarified.

\section{$\operatorname{Aim}$}

The aim of the study was to retrospectively compare $\mathrm{RD}$ responder patients to RD non-responders and find predictors of non-responsiveness for RD as well as clinical factors that might have a direct impact on the blood pressure reduction to increase the chances of procedural success.

\section{Material and methods \\ Patients and procedure}

All patients enrolled in the study were part of the HTN-1 and HTN-2 trials population. Adult patients with resistant arterial hypertension with systolic blood pressure over $160 \mathrm{~mm} \mathrm{Hg}$, receiving full doses of three antihypertensive drugs (including a diuretic) for at least 2 weeks before enrollment and with an estimated glomerular filtration rate over $45 \mathrm{ml} / \mathrm{min}$ who met all inclusion/exclusion criteria were eligible for this study. The presence of resistant arterial hypertension was defined as mean brachial artery seated systolic pressure above $160 \mathrm{~mm} \mathrm{Hg}$ during out-patient department visits, despite historical compliance with at least three antihypertensive drugs (including a diuretic) in optimal doses. Blood pressure measurement was performed in accordance with Joint National Committee VII guidelines [6]. Measurements were performed each time by the same person, sitting, in triplicate and then averaged. The technique of renal denervation has recently been described [7-10]. After standard femoral vascular access, the SIMPLICITY catheter (Ardian Inc. USA Company, currently Medtronic Inc., USA) was introduced into each renal artery. During the procedure, a standard dose of unfractionated heparin was used. Six radiofrequency ablations at $8 \mathrm{~W}$ lasting up to $120 \mathrm{~s}$ each were performed in both renal arteries. Supplied energy, tip temperature and impedance were monitored by the catheter system in response to a predetermined algorithm during the procedure. Follow-up visits were performed at 1, 3, 6, 9, 12, 18, 24 and 36 months after the index procedure. Blood pressure (systolic, diastolic, pulse pressure) measurements were performed according to the methodology described earlier. Blood count, electrolytes, urea, creatinine, urine and physical examinations including orthostatic BP measurements were performed. During the entire study major adverse events (death, stroke, myocardial infarction) or other outcomes associated with the procedure were monitored. Three years after the procedure we assessed the percentage reduction of the mean baseline blood pressure value. Patients were assigned to the "non-responders" group if the percentage reduction of systolic blood pressure was $\leq 10 \%$ than the baseline. Patients were assigned to the "responders" group if the percentage reduction of systolic blood pressure was at least $10 \%$ of mean systolic BP. Written informed consent was obtained from all patients. The study was approved by the institutional ethical board (two separate ethics committee approvals since the patients attended the SYMPLICITY HTN-1 and SYMPLICITY HTN-2 studies).

\section{Analysis}

1. Multiple regression analysis of all factors was performed to find any determinants of BP reduction.

2. The group of patients with BP reduction after RD (responders) was compared to the group of patients without BP reduction after RD (non-responders).

\section{Statistical analysis}

Continuous variables were checked for normal distribution with the Shapiro-Wilk test. Data are expressed as mean \pm standard deviation or median (interquartile range) unless otherwise stated. To assess the differences between two continuous variables, Student's $t$-test (for normally distributed values), or the Mann-Whitney $U$-test (for non-normally distributed values) was applied. To assess the differences between three continuous variables, the ANOVA test (for normally distributed values) or the Kruskal-Wallis-test (for non-normally distributed values) was applied. Categorical variables were compared by the $\chi^{2}$ test or Fisher's exact test, as appropriate. Multiple regression was calculated to assess the relationship between one dependent variable and one or more independent variables. Statistical analysis was performed with Statistica 10.0 (StatSoft, Tulsa, OK, USA). A two-sided $p$-value $<0.05$ was considered statistically significant.

\section{Results}

Before the procedure systolic BP from 160 to $170 \mathrm{~mm} \mathrm{Hg}$ was present in $35.5 \%$ of patients; systolic BP above 170 to $180 \mathrm{~mm} \mathrm{Hg}$ was present in $48.4 \%$ of patients; systolic BP above $180 \mathrm{~mm} \mathrm{Hg}$ was present in $16.1 \%$ of patients. Patients' characteristic are presented in Table I. After 3 years of follow-up a $10 \%$ or more reduction of systolic baseline BP was observed in $74 \%$ of patients. A $10 \%$ or more reduction of diastolic baseline BP was observed in $71 \%$ of patients. The mean value of sys- 
Table I. Baseline patients' characteristics

\begin{tabular}{lc} 
Variable & Value \\
\hline Sex (female) & $38.7 \%$ \\
\hline Age (mean) [years] & $31-72(57.3)$ \\
\hline BMI (mean) $\left[\mathrm{kg} / \mathrm{m}^{2}\right]$ & $23.36-46.81(33.0)$ \\
\hline Weight $[\mathrm{kg}]$ & $77-145(94.7)$ \\
\hline Height $[\mathrm{cm}]$ & $160-184(169.4)$ \\
\hline CAD & $24.1 \%$ \\
\hline Cardiomyopathy & $6.9 \%$ \\
\hline Ventricular arrhythmia & $6.9 \%$ \\
\hline Hypercholesterolaemia & $62.1 \%$ \\
\hline Diabetes mellitus type 2 & $31.0 \%$ \\
\hline COPD & $6.9 \%$ \\
\hline Obesity & $51.7 \%$ \\
\hline Renal insufficiency & $4.4 \%$ \\
\hline
\end{tabular}

Table II. Multivariate logistic regression analysis of risk factors in response patients group

\begin{tabular}{lccc} 
Variable & Odds ratio & $\begin{array}{c}\text { 95\% confidence } \\
\text { intervals }\end{array}$ & $\boldsymbol{P}$-value \\
\hline Age & 1.046 & $0.9405-1.1633$ & 0.4 \\
\hline Female sex & 3.0177 & $0.3565-25.5471$ & 0.31 \\
\hline CAD & 0.14 & $0.0146-1.3397$ & 0.08 \\
\hline COPD & 0.4217 & $0.0121-14.6716$ & 0.63 \\
\hline DM2 & 1.0904 & $0.0973-12.2245$ & 0.94 \\
\hline Obesity & 0.9879 & $0.1002-9.7389$ & 0.99
\end{tabular}

DM2 - diabetes mellitus type 2, CAD - coronary artery disease, COPD - chronic obstructive pulmonary disease.

tolic BP ranged from 110 to $211 \mathrm{~mm} \mathrm{Hg}$ (mean value: $149.69 \mathrm{~mm} \mathrm{Hg}$ ) and it was $25.23 \mathrm{~mm} \mathrm{Hg}$ lower than baseline BP. The mean percentage reduction was $14 \%$ in all patients. The mean value of diastolic BP ranged from 64 to $119 \mathrm{~mm} \mathrm{Hg}$ (mean value: $85.17 \mathrm{~mm} \mathrm{Hg}$ ) and it was $14.65 \mathrm{~mm} \mathrm{Hg}$ lower than baseline BP. The percentage reduction was $14 \%$. Before ablation the mean systolic $\mathrm{BP}$ value of three measurements ranged from 162 to $212 \mathrm{~mm} \mathrm{Hg}$ (mean value: $174.91 \mathrm{~mm} \mathrm{Hg}$ ). Target systolic $\mathrm{BP} \leq 140 \mathrm{~mm} \mathrm{Hg}$ was achieved in $35 \%$ and target diastolic BP $\leq 90 \mathrm{~mm}$ Hg was achieved in $71 \%$ of patients. Target systolic and diastolic $\leq 140 / 90 \mathrm{~mm} \mathrm{Hg}$ was achieved in $35 \%$ of patients (Figure 1). Relationships between percentage reduction of baseline systolic $\mathrm{BP}$ and risk factors are present in Table II. A 20\% reduction of baseline systolic blood pressure was observed in $35 \%$ of patients (high response patients). A reduction from 10\% to $20 \%$ of baseline systolic blood pressure was observed in $39 \%$

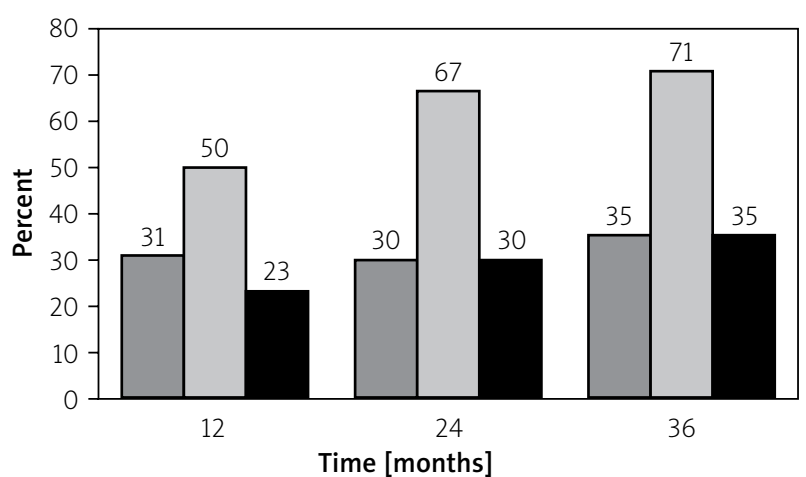

$\square$ Systolic BP $\leq 140 \mathrm{~mm} \mathrm{Hg} \quad \square$ Diastolic BP $\leq 90 \mathrm{~mm} \mathrm{Hg}$ - Systolic/diastolic BP $\leq 140 / 90 \mathrm{~mm} \mathrm{Hg}$

Figure 1. Percentage of patients who achieved target systolic, diastolic and systolic/diastolic blood pressure values 12,24 and 36 months after the procedure

$B P$ - blood pressure.

of patients (normal response patients). Twenty-six percent of patients had no response for renal denervation.

\section{Response patients $->10 \%$ baseline systolic $\mathrm{BP}$ reduction}

Twenty-two $(71 \%)$ patients had $>10 \%$ reduction of mean baseline systolic BP. There were 10 females and 12 males (45\% female and $55 \%$ male) in the response group. Patients' age ranged from 43 to 76 (mean value: 57.04 ), weight ranged from 74 to $122 \mathrm{~kg}$ (mean value: $94.7 \mathrm{~kg}$ ), height ranged from 159 to $184 \mathrm{~cm}$ (mean value: $169.3 \mathrm{~cm}$ ) and body mass index (BMI) ranged from 23.36 to $39.41 \mathrm{~kg} / \mathrm{m}^{2}\left(33.02 \mathrm{~kg} / \mathrm{m}^{2}\right)$.

Observed risk factors in this group were: hypercholesterolemia in $70 \%, \mathrm{BMI}>30 \mathrm{~kg} / \mathrm{m}^{2}$ in $55 \%$, diabetes mellitus in 35\%, current smoking in $5 \%$. Comorbidity included coronary artery disease (CAD) in 30\%, cardiomyopathy in $10 \%$, chronic obstructive pulmonary disease (COPD) in $10 \%$, renal insufficiency in $10 \%$, and ventricular arrhythmia in $5 \%$. Multivariate logistic regression analysis of risk factors in response patients is presented in Table II.

\section{Non-response patients}

Eight (26\%) patients did not respond to renal denervation. This group consisted of 2 females and 6 males (25\% female and $75 \%$ male). Patients' age ranged from 31 to 72 (mean value: 56.1), weight ranged from 83 to $145 \mathrm{~kg}$ (mean value: $99.14 \mathrm{~kg}$ ), height ranged from 160 to $176 \mathrm{~cm}$ (mean value: $168.86 \mathrm{~cm}$ ) and BMI ranged from 29.39 to $46.81 \mathrm{~kg} / \mathrm{m}^{2}\left(34.56 \mathrm{~kg} / \mathrm{m}^{2}\right)$. Observed risk factors in this group were: hypercholesterolemia in $38 \%$, diabetes mellitus type 2 in $38 \%$ and $\mathrm{BMI}>30 \mathrm{~kg} / \mathrm{m}^{2}$ in $86 \%$. Comorbidity included CAD in $50 \%$ and cardiomyopathy in $13 \%$ of patients.

Differences between clinical factors in regard to responders and non-responders are presented in Table III. 
Table III. Comparison of clinical factors between response patients and non-response patients

\begin{tabular}{|c|c|c|c|}
\hline Variable & Response patients & Non-response patients & $P$-value \\
\hline Sex (female) & $40.9 \%$ & $25.0 \%$ & 0.55 \\
\hline Age (mean) [years] & $43-76(57.0)$ & $31-72(56.1)$ & 0.32 \\
\hline BMI (mean) $\left[\mathrm{kg} / \mathrm{m}^{2}\right]$ & 23.36-39.41 (33.0) & 29.39-46.81 (34.6) & 0.94 \\
\hline Weight [kg] & $74-122(94.7)$ & 83-145 (99.14) & 0.71 \\
\hline Height & 159-184 (169.3) & 160-176 (168.86) & 0.70 \\
\hline CAD & $27.2 \%$ & $50.0 \%$ & 0.57 \\
\hline Cardiomyopathy & $9.1 \%$ & $12.5 \%$ & 0.66 \\
\hline Ventricular arrhythmia & $4.5 \%$ & $0 \%$ & 0.63 \\
\hline Hypercholesterolemia & $63.6 \%$ & $37.5 \%$ & 0.24 \\
\hline Diabetes mellitus type 2 & $55.50 \%$ & $37.5 \%$ & 0.75 \\
\hline COPD & $9.1 \%$ & $0 \%$ & 0.91 \\
\hline Obesity & $63.6 \%$ & $87.5 \%$ & 0.67 \\
\hline Current smoker & $4.5 \%$ & $0 \%$ & 0.69 \\
\hline CKD: & & & 0.13 \\
\hline CKD 1 & $50 \%$ & $50 \%$ & \\
\hline CKD 2 & $41.9 \%$ & $50 \%$ & \\
\hline CKD 3a & $9.1 \%$ & $0 \%$ & \\
\hline
\end{tabular}

$B M I$ - body mass index, CAD - coronary artery disease, CKD - chronic kidney disease, COPD - chronic obstructive pulmonary disease.

\section{Discussion}

Radiofrequency renal nerve ablation is effective treatment in patients with resistant hypertension by reducing the level of blood pressure $[1,2,8,10]$. Patients with resistant hypertension are at a higher risk of cardiovascular events and end-organ damage as compared to patients with adequately controlled hypertension [11]. The efficacy of the RD procedure has been assessed in several large studies. The Symplicity HTN-1 and randomized Symplicity HTN-2 trial demonstrated that RD is feasible, effective and safe in the treatment of resistant hypertension. In both studies, no adverse effects of RD on renal function were observed [1, 2]. However, the Symplicity HTN-1 and HTN-2 trials did not include only patients with bilateral single arteries. Further research showed that BP reduction can also be achieved in patients with accessory renal arteries [12]. The Symplicity HTN-3 trial suggested that 6 months after RD there were no significant differences in reduction of systolic BP in office or 24-hour ambulatory measurements as compared with a sham control [13]. The results of the Symplicity HTN-3 trial did not confirm the results of previous registries and trials with a potentially overestimated treatment effect; however, several limitations of the study design might have influenced the clinical outcomes $[14,15]$. On the other hand, Symplicity HTN-3 confirmed the safety of the RD procedure. Based on the results of individual analyses, we can conclude that the final effect of RD will depend on many factors and this procedure may not be suitable for all patients. Identifying predictors which make RD effective is important to select patients who will respond adequately to treatment. Due to important uncertainties about RD, more research is required to provide conclusive evidence for antihypertensive long-term effects, safety, clinical outcomes and quality of life (QoL) improvement after RD. The additional benefits of RD observed in other studies were reduction of left ventricle mass and improvement of diastolic function. These might have important prognostic implications for patients with resistant hypertension at high cardiovascular risk [16]. Other studies have suggested a reduction in heart rate over 6 months $[1,17]$, as well as a positive effect on renal function [18], glucose metabolism and insulin sensitivity after RD [19]. In the present study, 3 years after the procedure, the mean BP value remained successfully reduced $(>10 \%$ reduction of baseline BP) in $74 \%$ of cases for systolic BP (response patients) and in $71 \%$ of cases for diastolic BP. Thirty-four percent of patients had $>20 \%$ reduction of baseline systolic BP, $39 \%$ had a $10 \%$ to $20 \%$ reduction of baseline systolic BP and $26 \%$ had less than a $10 \%$ reduction or the mean systolic BP was higher than the baseline BP (non-response patients). After renal denervation the systolic BP reduction was highest in the female group 
compared to the male group. Women were more frequently in the group of response patients ( $49 \%$ women vs. $51 \%$ men) whereas men were more frequently in the group of non-response patients (25\% women vs. $75 \%$ men). It should be noted that female sex is associated with the highest risk of resistant hypertension [19]. In our study, renal denervation in women led to a $16 \%$ reduction $(28.25 \mathrm{~mm} \mathrm{Hg})$ and in men to a $13 \%$ reduction (23.28 $\mathrm{mm} \mathrm{Hg}$ ) of baseline systolic BP. There was a trend towards better responsiveness for RD in older and taller patients and non-responsiveness in obese patients. However, there was not significant in the formal statistical sense $(p<0.05)$. The prevalence of hypercholesterolemia was higher in response patients (64\%) compared to non-response patients (38\%). Hypercholesterolemia is not a risk factor for resistant hypertension, but it may lead to vascular disease such as atherosclerosis or renal artery stenosis which can cause resistant hypertension [20]. Moreover, systolic BP reduction is more difficult to achieve in older patients [20]. In our study, renal denervation in all hypercholesterolemia patients led to a $16 \%$ reduction (28.28 $\mathrm{mm} \mathrm{Hg}$ ) of baseline systolic BP. Prevalence of diabetes type II, commonly associated with hypertension, was higher in response patients (present in 55.5\%) compared to non-response patients (37.5\%). Increased levels of insulin resistance cause a proportional increase in severity of hypertension. Patients with increased sympathetic nervous activity and vascular smooth muscle cell proliferation present increased sodium retention [21]. Therefore, in diabetes patients, denervation of sympathetic nerves decreases the level of sodium retention. Also diabetic patients need more antihypertensive medications (range: 2.8-4.2) to achieve target BP compared to non-diabetic patients [22]. Preliminary studies have shown that renal denervation is associated with a reduction in fasting glucose and insulin levels [23]. In our study, renal denervation in all diabetes mellitus type 2 patients led to a $17 \%$ reduction $(29.41 \mathrm{~mm} \mathrm{Hg})$ of baseline systolic BP. Obesity was more frequent in the non-response group (present in $87.5 \%$ ) compared to response patients (63.6\%). Also, higher BMI values were present in non-response patients $\left(34.56 \mathrm{~kg} / \mathrm{m}^{2}\right)$ compared to response patients $\left(33 \mathrm{~kg} / \mathrm{m}^{2}\right)$. There were no significant differences between groups. Obesity is the main lifestyle risk factor for resistant hypertension [24] and with high BMI is associated with more severe hypertension and increased likelihood of never achieving optimal blood pressure [25]. It is assumed that pathophysiological increased activation of the sympathetic nervous system caused by obesity is one of the main reasons for resistant hypertension [26]. A recent study showed that renal denervation led to suppression of renal sympathetic nerve activity and reduction of total body noradrenaline $[1,26]$. In our study, renal denervation in all obese patients led to a $13 \%$ reduction (22.15 mm Hg) of baseline systolic BP. Coronary artery disease was more frequent in non-response patients (50\%) compared to response patients (27\%). A recent study suggested that renal denervation, despite BP reduction, also has beneficial effects on heart disease. Improvements in symptoms and exercise capacity in patients with chronic systolic heart failure were confirmed by Davies et al. [27]. Other benefits for the cardiovascular system after renal denervation are: improvement of cardiac diastolic function, reduction of left ventricular mass and reduction of the augmentation index [28, 29]. The theoretical potential of renal denervation in cardiac arrhythmia is currently being investigated [30].

$A$ recent study suggested that renal denervation, despite $B P$ reduction, also has beneficial effects on heart disease like the improvement of cardiac diastolic function, reduction of left ventricular mass, reduction of the augmentation index and exercise capacity [21, 29, 30]. The theoretically potential of renal denervation on cardiac arrhythmia is currently investigated [30].

Renal insufficiency was observed only in high response patients (18\%). Renal diseases are a common cause of resistant hypertension [26]. Renal denervation is a potential therapeutic option for hemodialysis patients with resistant or difficult to control hypertension. In our study, renal denervation in renal insufficiency patients led to a $22 \%$ reduction $(22.93 \mathrm{~mm} \mathrm{Hg}$ ) of baseline systolic BP.

Resistant hypertension is a specific disease with complex etiology often comorbid with other diseases that require multidisciplinary treatment. Denervation (ablation) of sympathetic fibers in renal arteries is an effective and safe treatment in resistant hypertensive patients, as has been proven by numerous clinical studies. However, there is a group of patients who do not respond to treatment. Nevertheless, there are numerous groups of patients with comorbid disease in whom renal denervation brings a number of additional therapeutic and clinical benefits. In our opinion, thoughtful selection of patients' enrollment in the ablation procedure will further enhance the benefits of the procedure and extend the range of indications for renal denervation. Due to the small number of patients in our analysis, a multicenter study should be performed for more detailed characteristics of patients who underwent renal denervation.

\section{Conclusions}

A $10 \%$ reduction of systolic baseline BP was observed in $74 \%$ of patients three years after renal denervation. Clinical factors like COPD, chronic kidney disease $3 \mathrm{a}$, female sex and hypercholesterolemia increase the chances of effective reduction of BP. However, due to the small number of patients, there was no statistically significant difference between these factors.

\section{Conflict of interest}

The authors declare no conflict of interest. 


\section{References}

1. Krum H, Schlaich $M$, Whitbourn R, et al. Catheter-based renal sympathetic denervation for resistant hypertension: a multicentre safety and proof-of-principle cohort study. Lancet 2009; 373: 1275-81.

2. Esler MD, Krum H, Sobotka PA, et al. Renal sympathetic denervation in patients with treatment-resistant hypertension (The Symplicity HTN-2 Trial): a randomised controlled trial. Lancet 2010; 376: 1903-9.

3. Collins R, Peto R, MacMahon S, et al. Blood pressure, stroke, and coronary heart disease. Part 2. Short-term reductions in blood pressure: overview of randomised drug trials in their epidemiological context. Lancet 1990; 335: 827-38.

4. Turnbull F. Effects of different blood-pressure-lowering regimens on major cardiovascular events: results of prospectively-designed overviews of randomised trials. Lancet 2003; 362: 1527-35.

5. Krawczyk-Ozog A, Tokarek T, Moczala K, et al. Long-term quality of life and clinical outcomes in patients with resistant hypertension treated with renal denervation. Adv Interv Cardiol 2016; 12: 329-33.

6. Muntner P, Krousel-Wood M, Hyre AD, et al. Antihypertensive prescriptions for newly treated patients before and after the main antihypertensive and lipid-lowering treatment to prevent heart attack trial results and seventh report of the joint national committee on prevention, detection, evaluation, and treatment of high blood pressure guidelines. Hypertension 2009; 53: 617-23.

7. Bartus K, Sadowski J, Kapelak B, et al. Denervation of nerve terminals in renal arteries: one-year follow-up of interventional treatment of arterial hypertension. Kardiol Pol 2014; 72: 425-31.

8. Bartus K, Sadowski J, Kapelak B, et al. Denervation (ablation) of nerve terminalis in renal arteries: early results of interventional treatment of arterial hypertension in Poland. Kardiol Pol 2013; 71: 152-8.

9. Esler MD, Bohm M, Sievert $H$, et al. Catheter-based renal denervation for treatment of patients with treatment-resistant hypertension: 36 month results from the SYMPLICITY HTN-2 randomized clinical trial. Eur Heart J 2014; 35: 1752-9.

10. Bartus K, Sadowski J, Kapelak B, et al. Renal denervation - interventional radiofrequency treatment of arterial hypertension. Two years follow-up. Kardiol Pol 2017.

11. Januszewicz A, Witkowski A. Renal denervation - where do we stand in 2014? Postep Kardiol Interw 2014; 10: 1.

12. Bartus K, Podolec J, Sadowski J, et al. Blood pressure reduction in patients with accessory renal arteries and bilateral single renal arteries after catheter-based renal denervation: prospective study with 3-year follow-up. Pol Arch Intern Med 2017; 127: 423-8.

13. Bhatt DL, Kandzari DE, O'Neill WW, et al. A controlled trial of renal denervation for resistant hypertension. N Engl J Med 2014; 370: 1393-401.

14. Warchoł-Celińska E, Januszewicz A, Prejbisz A, et al, Renal denervation after the symplicity HTN-3 trial. Postep Kardiol Interw 2014; 10: 75-7.

15. Luscher TF, Mahfoud F. Renal nerve ablation after SYMPLICITY HTN3: confused at the higher level? Eur Heart J 2014; 35: 1706-11.

16. Brandt MC, Mahfoud F, Reda S, et al. Renal sympathetic denervation reduces left ventricular hypertrophy and improves cardiac function in patients with resistant hypertension. J Am Coll Cardiol 2012; 59: 901-9.
17. Ukena C, Mahfoud F, Spies A, et al. Effects of renal sympathetic denervation on heart rate and atrioventricular conduction in patients with resistant hypertension. Int J Cardiol 2013; 167: 2846-51.

18. Mahfoud F, Cremers B, Janker J, et al. Renal hemodynamics and renal function after catheter-based renal sympathetic denervation in patients with resistant hypertension. Hypertension 2012; 60: 419-24.

19. Schlaich MP, Straznicky N, Grima M, et al. Renal denervation: a potential new treatment modality for polycystic ovary syndrome? J Hypertens 2011; 29: 991-6.

20. Sarafidis PA, Bakris GL. Resistant hypertension: an overview of evaluation and treatment. J Am Coll Cardiol 2008; 52: 1749-57.

21. Nishizaka MK, Pratt-Ubunama M, Zaman MA, et al. Validity of plasma aldosterone-to-renin activity ratio in African American and white subjects with resistant hypertension. Am J Hypertens 2005; 18: 805-12.

22. Bakris GL. A practical approach to achieving recommended blood pressure goals in diabetic patients. Arch Intern Med 2001; 161: 2661-7.

23. Hering D, Esler MD, Schlaich MP. Effects of renal denervation on insulin resistance. Expert Rev Cardiovasc Ther 2012; 10: 1381-6.

24. Bramlage, P, Pittrow D, Wittchen HU, et al. Hypertension in overweight and obese primary care patients is highly prevalent and poorly controlled. Am J Hypertens 2004; 17: 904-10.

25. Hall JE. The kidney, hypertension, and obesity. Hypertension 2003; 41: 625-33.

26. Schlaich MP, Hering D, Sobotka P, et al. Effects of renal denervation on sympathetic activation, blood pressure, and glucose metabolism in patients with resistant hypertension. Front Physiol 2012; 3: 10.

27. Davies JE, Manisty CH, Petraco R, et al. First-in-man safety evaluation of renal denervation for chronic systolic heart failure: primary outcome from REACH-Pilot study. Int I Cardiol 2013; 162: 189-92.

28. Hering D, Lambert EA, Marusic P, et al. Renal nerve ablation reduces augmentation index in patients with resistant hypertension. J Hypertens 2013; 31: 1893-900.

29. Qiu M, Yin Y, Shan Q. Renal sympathetic denervation versus antiarrhythmic drugs for drug-resistant hypertension and symptomatic atrial fibrillation (RSDforAF) trial: study protocol for a randomized controlled trial. Trials 2013; 14: 168.

30. Ukena C, Mahfoud F, Linz D, et al. Potential role of renal sympathetic denervation for the treatment of cardiac arrhythmias. Eurolntervention 2013; 9 Suppl R: R110-6. 